\title{
The effectiveness of sustainable serial casting for clubfoot deformity in a low resource setting
}

\author{
James Turner ${ }^{1}$, Freddie Quiney ${ }^{2}$ John Cashman ${ }^{3}$, Chris Lavy ${ }^{4}$ \\ 1. CURE Children's Hospital Malawi \\ 2. University of Oxford (fredquiney@homtail.co) \\ 3. Sheffield Children's Hospital, Trauma and Orthopaedics (Jpcashman@gmail.com) \\ 4. University of Oxford (Chris.lavy@ndorms.ox.ac.uk)
}

Corresponding author: James Turner; jimturner@doctors.org.uk

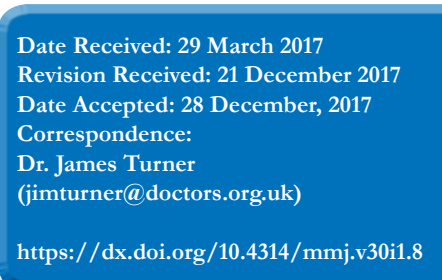

Abstract

Aims

Our aim was to assess the effectiveness of the Malawi Clubfoot Programme and comment on such health care strategies in developing countries.

Methods

Medical records of 1069 children attending 29 clinics within Malawi were identified between 2007 and 2013. Due to incomplete recording, only 596 patients had adequate data which could be further analysed.

Results

The mean age of presentation was 103 days (range $0-8$ years) with a sex distribution of M1.76:F1. The mean Pirani score at presentation was 4.55 and on completion of casting was 1.39. A correlation was identified between the number of castings and the initial Pirani score (positive coefficient $0.2626 \mathrm{p}<0.0001$ ), the final casting score (negative coefficient $-0.1441 \mathrm{p}<0.0006$ ) and the change in Pirani score (positive coefficient $0.3200(\mathrm{p}<0.0001)$ ). The number of patients attending the clinics increased per year and the average number of castings was reduced from 6 to 5 between 2008 and 2012. There was also moderate correlation between the number of years the programme had been re-instated and the average change in Pirani score between in each casting (R score 0.36)

Conclusion

Serial casting performed by paramedical personnel within an established self- sustained national programme can effectively treat CTEV in low resource countries.

\section{Introduction}

Congenital talipes equinovarus is a curable congenital abnormality affecting the foot and ankle. The deformity is most commonly idiopathic but secondary congenital talipes equinovarus does exist. In North America and Europe, the incidence of idiopathic club foot is $1 / 1000$ with $50 \%$ of cases being bilateral ${ }^{1}$. However, this rate is substantially higher in other parts of the world; a recent study has estimated an incidence of 2/1000 in Malawi, one of the highest rates worldwide ${ }^{2}$. This translates to over 1200 new cases being diagnosed each year and in a country with one of the lowest Gross Domestic Product (GDP), a significant burden is placed on its financial, medical and social services. The current gold standard treatment is the Ponseti method, using a protocol that involves serial casting from the point of identification within the neonatal period $^{3}$.

The Ponseti method was introduced in Malawi in 2001 and a national programme with a series of clinics was set up, but there was little formal support in terms of supplies and continuing training. This led to a re-launch of the programme in 2007 with managerial assistance from the Non-Governmental Organisation, CURE International.

The primary goal of the programme is to ensure that every child born in Malawi with the condition is treated appropriately using the method of serial casting and to eradicate cases of untreated clubfoot. There are 26 clinics which have been strategically placed in areas across the country where no one individual has to travel for more than one day to receive assessment and treatment. Local healthcare providers within district hospitals and clinics have been trained to identify and manage the deformity. In this study, we have collected and analysed data from the Malawi Clubfoot Programme re-launched in 2007 and we report the efficacy of such a healthcare improvement strategy in subSaharan Africa.

\section{Patients And Methods}

We visited all 26 clinics of the Malawi Clubfoot Programme and we were able to identify the records of 1069 children who have been treated for CTEV between January 2007 and August 2013. The precise diagnosis, severity, stages of treatment and outcomes were recorded on a standardised form by the clinician at the clinic and later analysed at the central co-ordinating centre in Blantyre.

\section{Results}

Due to incomplete demographic data recording, a total of 335 patients had to be omitted from the study. A further 138 patients were lost to follow up where an initial Pirani score was recorded but no further scores were recorded. This meant that a final number of 596 subjects had both scores recorded (340 bilateral, 113 left and 143 right; ratio of bilateral: unilateral of 1.32: 1). So a total of 936 castings were analysed from which our conclusions were drawn. For the 596 patients, the mean age of presentation was 97 days (95\% C.I. 75.8 to 117.9 ) with a range of 0 days to 8 years. The gender distribution was male: female ratio of 1.77:1.

Pirani score was recorded at the start of casting and on cast completion. The mean casting score on presentation was 4.55 and the mean casting score on completion of casting was 1.39. This gave a mean difference of 3.15 (95\% confidence 
interval of this difference from 3.004 to 3.302). A paired $\mathrm{t}$-test was used to determine if this difference in means was significant. With a $\mathrm{P}$ value of $<0.0001$, this difference has high statistical significance.

Data was also used to investigate correlations between a number of the variables:

- Correlation between initial casting score and number of castings: Analysis produced a correlation coefficient of 0.2626 ( $\mathrm{p}$ value of <.0001), thus a weak positive correlation which is highly significant. This suggests that a high initial Pirani score means the patient is more likely to have an increased number of castings.

- Correlation between final casting score and number of castings: Analysis produced a correlation coefficient of -0.1441 ( $\mathrm{p}$ value of 0.0006 ), thus a weak negative correlation which is highly significant. This suggests that those with a low final Pirani score were more likely to have had an increased number of castings.

- Correlation between change in Pirani score and number of castings: Analysis produced a correlation coefficient of 0.3200 ( $\mathrm{p}$ value of <.0001), thus the strongest of the correlations that were measured. This shows that, generally, those who had the greatest change in Pirani score also had the greatest number of castings.

We also studied, in detail, the effect of time on patient numbers and outcome measures; the number of feet treated per year has increased steadily, with annual cases more than doubling (Figure 1).

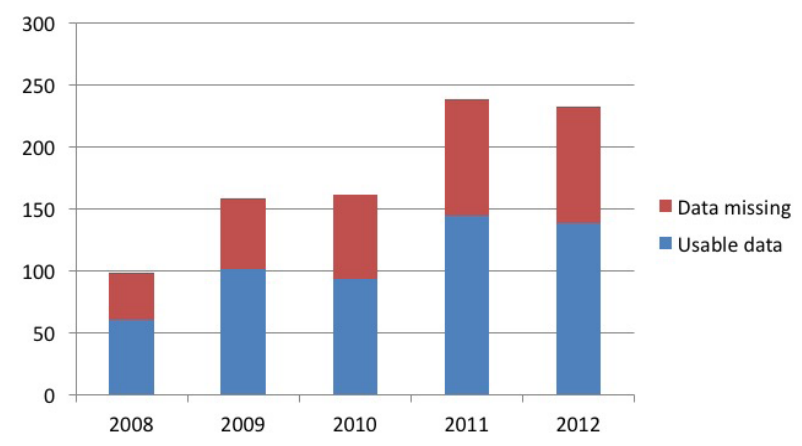

Figure 1: Total number of clubfeet seen each year

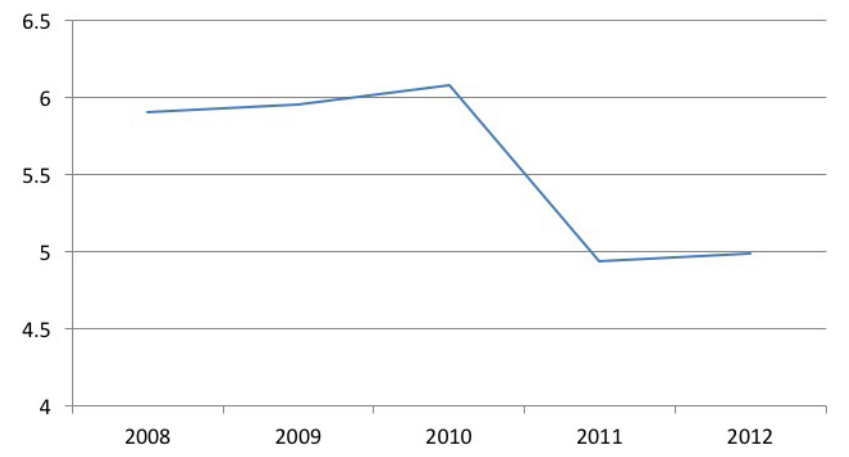

Figure 2: Average number of casts used to correct defomity

Figure 1: We found that, between 2008 and 2012, the average number of castings per individual dropped from 6 to 5 but this decrement was not statistically significant (figure 2). Figure 2: In addition, the average change in Pirani score between each casting had increased with time; a linear regression was performed on the data (figure 3) and with an R score of 0.36 , there is moderate correlation between https://dx.doi.org/10.4314/mmj.v30i1.8 year and score improvement. The $\mathrm{R}$ squared value of 0.13 shows that the change in year is responsible for $13 \%$ of the improvement in the Pirani scoring.

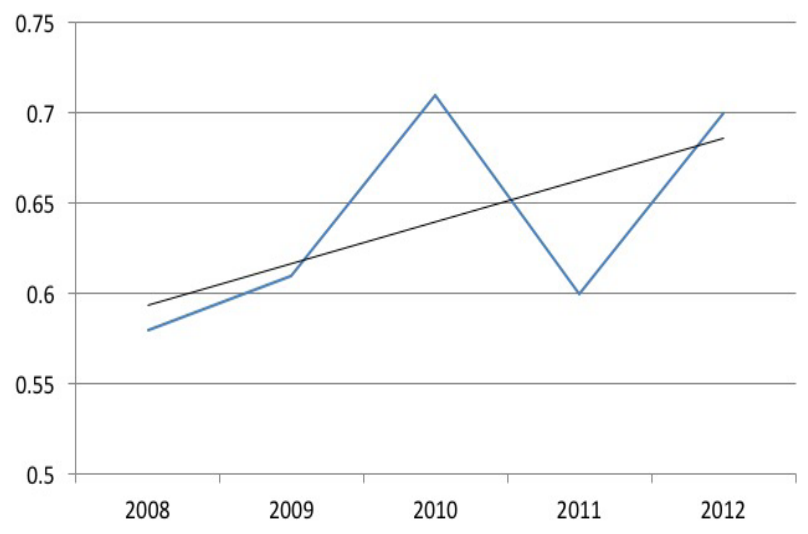

Figure 3: Improvement of Pirani score per casting

\section{Discussion}

The effectiveness of the Ponseti method was first examined in Malawi in 2003 when a review of the initial national programme showed a significant reduction in the number of cases that required surgery to correct the deformity ${ }^{3}$. It showed that 327 of the 482 cases $(68 \%)$ were reported to have reached plantigrade position. The reasons for this relatively low success rate included poor supplies of Plaster of Paris in some clinics so that treatment could not continue. A more recent analysis of serial casting in India has shown a success rate of $94 \%{ }^{4}$. Two further publications from Bangladesh and Zimbabwe support this positive finding ${ }^{5,6}$.

These four studies carried out in Low and Middle Income Countries suggest that sub-Saharan Africa would benefit from the introduction of a clubfoot programme and that this can be achieved by paramedical staff performing the manipulations. A prospective survey looking at the ability of orthopaedic clinical officers (non-medically trained trauma and musculo-skeletal clinicians) to correct clubfoot deformity found that 98 of 100 consecutive uncomplicated CTEVs were successfully manipulated by this group of clinicians ${ }^{7}$.

Due to scarcity of fully trained doctors in Malawi and the simplicity of the Ponseti method, it was decided that this group of healthcare workers would provide the most efficient service. A minimum of 3 care workers per district were trained. Foot splints and plaster cast material were provided by the programme co-ordinators and district health authorities. Recruitment to the clinic was by word of mouth, via maternity nursing staff and media advertising on $\mathrm{TV}$, radio and newspapers. Only 20 of the 25 clinics were functioning at the end of the pilot study. The healthcare workers in the districts without cover stated that there was no requirement for the programme, probably due to the children with clubfeet receiving treatment elsewhere. Our study did not document who treated which clubfeet so we cannot comment on the effectiveness of trained non-physicians compared with their medical counterparts, although we do know that the vast majority of the clinics throughout the country are run by para-medical staff.

In 2003, an estimated 1125 children were born with clubfoot but only $30 \%$ (342) of the expected cases presented to the clinics $^{3}$. Of the 342 seen, only 307 had adequate records. The mean age calculated was 75 days with 193 cases being male 
and 114 being female (1.7:1). 482 clubfeet were identified with 175 children presenting with bilateral deformities and 132 with unilateral clubfoot (1.3:1). The ratios of sex and effected side are very similar with those calculated in our study (male: female ratio 1.8:1 and bilateral: unilateral ratio 1.3:1) although the mean age at presentation throughout the existence of Malawi Clubfoot Programme has risen from 75 days to 103 days. This could be explained by the fact that once a healthcare improvement strategy has been established, all children with the pathology will be encouraged to attend the clinics, including those whose disease has been neglected for years.

This study recorded 1069 affected individuals who attended clinic since the re-establishment of the Malawi Clubfoot Programme in 2007. This translates to an average of 178 new patients per year and falls well short of the predicted $1200^{2}$. Our investigation did not set out to identify the causes of such a discrepancy so the methods of data collection and the precise data captured do not and cannot provide an explanation. However, possible causation from anecdotal evidence includes missing notes, inadequate data collection, insufficient public education and promotion of the programme and costs incurred during travelling to the clinics. A systemic review published in 2017 looked into the barriers of delivering an effective clubfoot service in LMICs and the solutions implemented ${ }^{8}$. Obstacles with the biggest impact included financial constraints, transportation, plaster and brace availability plus their care and fitting, providers lack of knowledge or skill and the health status perceived by the patients and their caregivers. Mentioned common solutions included financial aid, educating the provider and patient, setting up clinics near population centres, as well as establishing partnerships with clubfoot organisations. Despite the low capture rate in our study, there has been a steady increase each year (figure 1), providing evidence that a healthcare strategy programme in Africa is effective and sustainable. As previously mentioned, there is evidence to support the effectiveness of non-medically trained healthcare workers treating the deformity.

Throughout treatment, the drop-in Pirani score averaged 3.15 with a final score of 1.39 . These results illustrate that Ponseti casting is highly effective at treating Clubfeet in a third world country. It also further backs-up the evidence that trained doctors are not the only group of health-care workers that can successfully manage such an affliction. Although expected, feet with the highest initial score also required more castings. This finding is supported by the literature 9 . Figures 2 and 3 depict an improvement in the effectiveness of casting as the programme has become more established and the healthcare workers have improved their casting techniques. An R squared value of 0.13 is not very high but the range of clinical presentations is large and the linear progression shows a clearly positive trend.

Our study has supported the literature that CTEV can be treated effectively with serial casting in low resource countries and that the development of a well-supported national programme does increase the number of those with the deformity receiving treatment, in addition to improving the outcome. The strength of the study and the conclusions drawn are limited by its retrospective nature, the low proportion of children estimated to have clubfoot being managed by serial casting and therefore a low number of subjects being enrolled in the study, and a large percentage of individuals whose data was incomplete. Although a systemic review has been published8, further research would help to identify and explain the reasons behind the low capture rate which would enable the healthcare strategy to evolve and further reduce the incidence of children living with this deformity. Comparing the outcome of clubfeet managed by non-medical personnel and those managed by physicians in this setting would also provide more evidence to support the notion that a medical degree is not required to effectively treat the deformity.

\section{Acknowledgments}

The authors would like to thank Professor Richard McManus at Oxford University for his statistical help with this study.

\section{References}

1. Wallander H, Hovelius L, Michaelsson K. Incidence of congenital clubfoot in Sweden. Acta Orthop 2006;77-6:847-52.

2. Mkandawire NCK, E. Incidence and patterns of congenital talipes equinovarus (clubfoot) deformity at Queen Elizabeth Central Hospital, Blantyre, Malawi. ECAJS 2004;9-2:28-31.

3. Lavy CB, Mannion SJ, Mkandawire NC, Tindall A, Steinlechner C, Chimangeni $\mathrm{S}$ et al. Club foot treatment in Malawi - a public health approach. Disabil Rehabil 2007;29-11-12:857-62.

4. Gupta A, Singh S, Patel P, Patel J, Varshney MK. Evaluation of the utility of the Ponseti method of correction of clubfoot deformity in a developing nation. Int Orthop 2008;32-1:75-9.

5. Evans AM, Chowdhary MM, Kabir MH, Rahman MF. Walk for life the National Clubfoot Project of Bangladesh: the four-year outcomes of 150 congenital clubfoot cases following Ponseti method. J Foot Ankle Res 2016;9:42.

6. Smythe T, Chandramohan D, Bruce J, Kuper H, Lavy C, Foster A. Results of clubfoot treatment after manipulation and casting using the Ponseti method: experience in Harare, Zimbabwe. Trop Med Int Health 2016;21:1311-1388.

7. Tindall AJ, Steinlechner CW, Lavy CB, Mannion S, Mkandawire N. Results of manipulation of idiopathic clubfoot deformity in Malawi by orthopaedic clinical officers using the Ponseti method: a realistic alternative for the developing world? J Pediatr Orthop 2005;25-5:6279.

8. Johnson RR, Friedman JM, Becker AM, Spiegel DA. The Ponseti Method for Clubfoot Treatment in Low and Middle-Income Countries: A Systematic Review of Barriers and Solutions to Service Delivery. J Pediatr Orthop. 2017;37:e134-e139.

9. Agarwal A, Gupta N. Does initial Pirani score and age influence number of Ponseti casts in children? Int Orthop 2014;38-3:569-72. 